\title{
CO IN THE MILKY WAY
}

\author{
LEO BLITZ \\ Astronomy Department \\ University of Maryland \\ College Park, MD 20742 USA
}

\begin{abstract}
If the CO distribution of the Milky Way is described as a truncated exponential rather than as a molecular ring with some gas at large radii, it becomes easier to understand the evolution of the disk of stars. The star formation rate per unit molecular gas mass is constant as a function of radius, and the $\mathrm{H}_{2}$ depletion time turns out to be only a few percent of the Hubble time. This very short timescale requires that the atomic gas act as a reservoir for the active star forming gas. Because the HI has such a different radial distribution, there must either be infall from outside the Galaxy, an efficient way for the atomic gas in the disk to lose angular momentum, or both, leading to measurable infall or inflow velocities. The truncation radius of $\mathrm{CO}$ is probably due to the recently identified stellar bar.
\end{abstract}

\section{Introduction}

The ubiquity of CO in the Milky Way was noticed soon after the discovery paper by Wilson et al. (1970) and it was shortly thereafter that CO surveys of different kinds of objects in the Milky Way (Wilson et al. 1974) and the Milky Way itself (Schwartz et al. 1972) were attempted. The difficulty of making an accurate CO survey of the Galactic plane soon became apparent, however, and it was not until the late 1970s that the first truly successful CO surveys were made (e.g., Cohen 1977; Burton \& Gordon 1978, Sanders et al. 1984). Figure 1 below shows the radial $\mathrm{CO}$ distribution (plotted as $\mathrm{H}_{2}$ mass surface density) from the most completely sampled data set, which was made with the $1.2 \mathrm{~m}$ sky survey telescope now located at the Center for Astrophysics (from Dame 1993). Also shown is the radial distribution of HI using two different outer Galaxy rotation curves. The figure succinctly points out what has been known since the mid-1970s: the distribution of 
molecules is very different from that of the atomic gas. The surface density ratio between the two phases varies by more than an order of magnitude between 0.5 and $2 \mathrm{R}_{\odot}$. Figure 1 also shows the large $\mathrm{H}_{2}$ surface density between 3 and $7 \mathrm{kpc}$ known as the "molecular ring." $\mathrm{R}_{\odot}$ is taken to be 8.5 kpc.

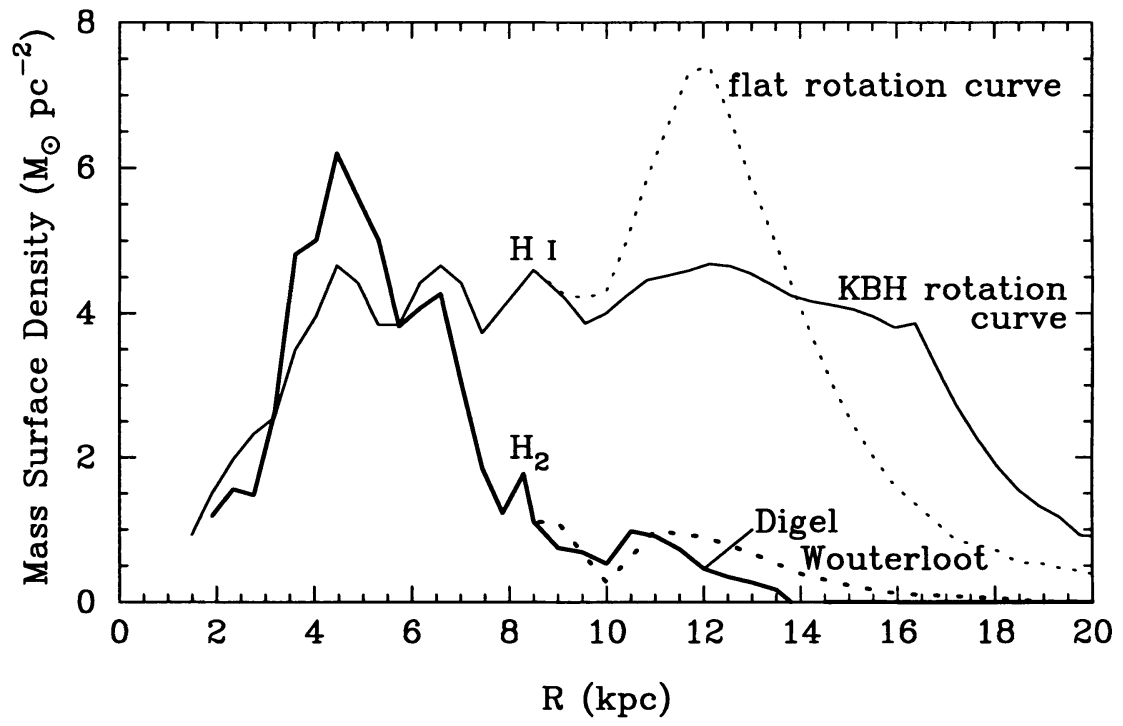

Figure 1. Plot of $\mathrm{H}_{2}$ and $\mathrm{HI}$ surface density as a function of Galactic radius from Dame (1993). The $\mathrm{H}_{2}$ surface density is measured as CO surface density multiplied by a constant conversion factor as described in the text; a recent recalibration lowers the $\mathrm{H}_{2}$ curve from that shown here by $\sim 25 \%$. See Dame 1993 for further details.

The conversion from $\mathrm{CO}$ emissivity to $\mathrm{H}_{2}$ surface density uses the wellknown "X-factor" (Bloemen et al. 1986), which relates the integrated CO line strength $T_{\mathrm{A}} d v$ to $N\left(\mathrm{H}_{2}\right)$. The value of $X$ used in Figure 1 is $2.3 \times 10^{20}$ $\mathrm{cm}^{-2}\left(\mathrm{~K} \mathrm{~km} \mathrm{~s}^{-1}\right)^{-1}$; recent recalibration of the $\mathrm{X}$-factor using GRO data suggests that a better value is $1.7-1.9 \times 10^{20} \mathrm{~cm}^{-2}\left(\mathrm{~K} \mathrm{~km} \mathrm{~s}^{-1}\right)^{-1}$ (Strong \& Mattox 1996). The lower value would decrease the amplitude of the $\mathrm{H}_{2}$ curve in Figure 1 by $25 \%$, implying that the $\mathrm{H}_{2}$ surface density is everywhere less than that of the atomic gas. The total mass of $\mathrm{H}_{2}$ in the Milky Way is $1.3 \times 10^{9} \mathrm{M}_{\odot}$ if the larger X-factor is used (Dame 1993) and $1.0 \times 10^{9} \mathrm{M}_{\odot}$ using the lower value. This compares to a total HI mass of about $5 \times 10^{9}$ $\mathrm{M}_{\odot}$ for HI integrated out as far as it is detected (Henderson et al. 1982; Wouterloot et al. 1990). Neither value includes the associated helium. 


\section{A Truncated Exponential}

Why does the CO distribution have the form that it does? Why is it so profoundly different from the HI? The difference is mirrored in the $\mathrm{CO}$ and HI distributions in all spiral galaxies and its origin provides a key to understanding the structure and evolution of disk galaxies in general. Part of the key is in recognizing that "molecular ring" is a misnomer. If one plots the $\mathrm{CO}$ distribution on a semi-log scale (see Figure 2), it is apparent that an exponential distribution is a good description of the radial dependence. The CO distribution is then more appropriately described as a truncated exponential rather than a ring; there is indeed nothing special about any part of the distribution outside the truncation radius of $\sim 3 \mathrm{kpc}$. The question then is what has removed the gas from within a radius of $4 \mathrm{kpc}$, rather than why is there so much gas between 3 and $7 \mathrm{kpc}$.

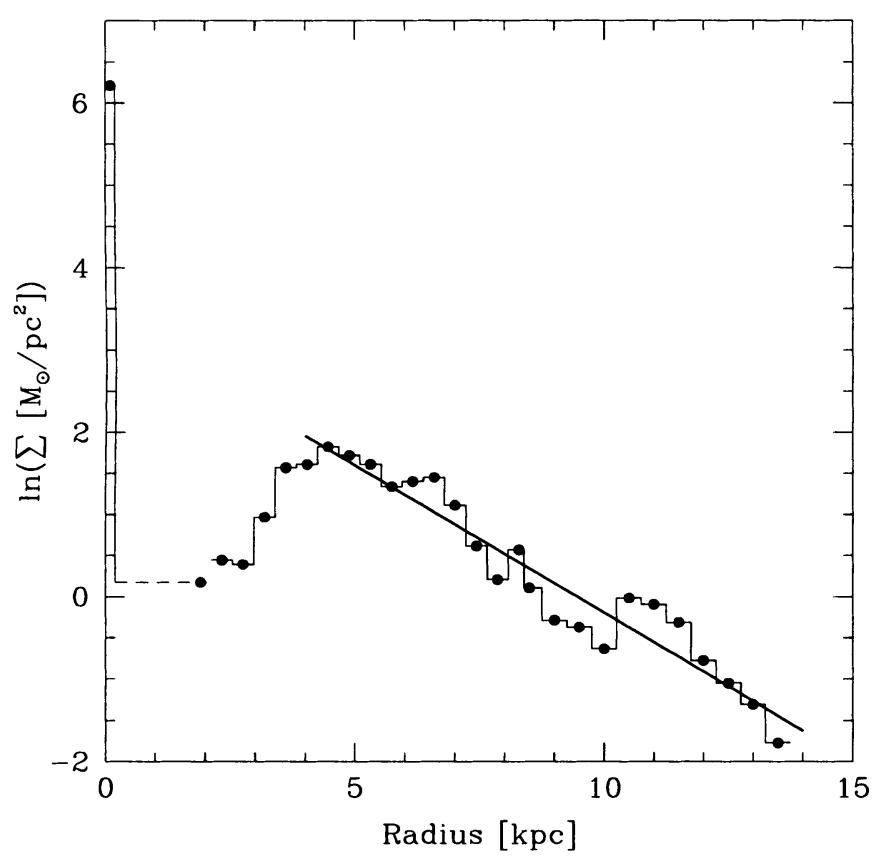

Figure 2. The $\mathrm{H}_{2}$ data from the curve marked Digel in Figure 1 with a least squares fit to the data at $R>4 \mathrm{kpc}$. The innermost point is based on data from Dame et al. 1987.

The scale length of the $\mathrm{CO}$ distribution in Figure 2 is $2.8 \mathrm{kpc}$. This is in good agreement with the scale length determined for all of the other tracers of current and past star formation. In a recent paper summarizing the distribution of various components of the disk (Prantzos \& Aubert 1995), 
the distributions of radio HII regions (Güsten \& Mezger 1983) and pulsars (Lyne et al. 1985) are found to have an exponential radial distribution with a scale length of about $3 \mathrm{kpc}$, similar to that of the CO. This result is not surprising; because the $\mathrm{CO}$ bearing gas is the progenitor of these population I objects, one would expect that they would all have the same radial distribution. However, an analysis of the COBE near infrared data (Spergel et al. 1996), which traces primarily much older stars, also obtains a good fit with an exponential disk of $3 \mathrm{kpc}$ scale length. The clear implication is that the Galactic distribution of currently forming stars echoes what has been taking place for the past several Gyr.

However, this raises an intriguing question. Does the distribution of star-forming gas that we see in the Milky Way reflect the gravitational potential determined by the radial stellar component, or is it the other way around? Is it the distribution of gas that determines the stellar distribution in the Milky Way and consequently in the disks of all spiral galaxies?

\section{Gas Depletion Times}

Some insight into this question comes from looking at the star formation rates in the Milky Way and the rate at which gas is returned to the ISM. Let us take the $\mathrm{H}_{2}$ mass of the Milky Way as $10^{9} \mathrm{M}_{\odot}$. There have been a number of estimates of the star formation rate, but a reasonably reliable one comes from Miller \& Scalo (1979) as part of their determination of the IMF. They obtain a local star formation rate of $3-7 \times 10^{-9} \mathrm{M}_{\odot} \mathrm{pc}^{-2}$ $\mathrm{yr}^{-1}$, which translates to a global star formation rate in the Milky Way of $2-4 \mathrm{M}_{\odot} \mathrm{yr}^{-1}$. Because all star formation results from the conversion of molecular gas into stars, the molecular gas would be depleted in $2.5-5$ $\times 10^{8} \mathrm{yr}$, only a few percent of the Hubble time! Furthermore, because the length scale of the star formation is the same as that of the molecular gas, the depletion timescale is independent of Galactic radius. Could it be, on the other hand, that the return of gas to the ISM from dying stars substantially makes up for the molecular depletion? Since the stellar distribution is already exponential, if the gas return could efficiently be transformed into molecules, it could explain the exponential gas distribution.

There are four broad classes of stars that return gas to the ISM: ordinary evolved stars in the AGB phase, massive stars giving rise to high velocity winds, supernovae, and main sequence stars producing winds similar to the solar wind. The return to the ISM has been estimated by various authors and is summarized in the table below. These numbers are for total mass returned to the ISM, rather than just hydrogen and helium mass, and are thus upper limits to the mass available for future star formation. Even if any one of these values has been significantly underestimated, the rate at 
which existing molecular gas is turned into new stars is $1-3 \mathrm{M}_{\odot} \mathrm{yr}^{-1}$, insubstantially changing the depletion time for the molecular gas.

TABLE 1. Gas Replenishment Rates

\begin{tabular}{lll}
\hline Source Type & $\mathrm{M}_{\odot} \mathrm{yr}^{-1}$ & Ref. \\
\hline Evolved Stars & 0.55 & Knapp (1991) \\
Massive Steller Winds & $\sim 0.2$ & Bieging (1989) \\
Supernovae & $\sim 0.02$ & van Buren (1985) \\
Main Sequence Stars & negligible & \\
Total & 0.8 & \\
\hline
\end{tabular}

\subsection{ATOMIC HYDROGEN}

Although the reservoir of molecular gas is relatively small, the reservoir of atomic gas is about 5-10 times larger. If all of the atomic gas were available for conversion into molecular gas, the gas depletion time could be increased to $1.5-5 \times 10^{9} \mathrm{yr}$, about $10-50 \%$ of the Hubble time, a value that is no longer implausibly short. However, because the distribution of atomic hydrogen is so different from that of the molecular gas (both in the Milky Way and most normal spirals), the gas depletion time near the truncation radius of the molecular gas remains disturbingly short, only about twice the value obtained from consideration of the molecular gas alone, or about 5 $10 \times 10^{8} \mathrm{yr}$, even if all of the atomic gas in that region were converted into stars. It seems that the only way around this dilemma is to require that there be a net inflow or infall of atomic gas, a conclusion reached some years back by Lacey and Fall (1985) from an analysis of the star formation history of the disk of the Milky Way. But if the molecular gas is replenished by inflow through the disk, there must be a process that efficiently transfers angular momentum of the gas outward. Furthermore, this process must produce a relative motion between the atomic and molecular gas to account for their different radial distributions.

A rough estimate of the inflow velocity can be obtained by requiring $10^{9}$ $\mathrm{M}_{\odot}$ of atomic gas to replenish the molecular gas inward of $2 \mathrm{R}_{\odot}$ in $5 \times 10^{8}$ yr. In this case, gas must travel a mean distance of $4 \mathrm{kpc}$, the distance outside the solar circle within which there is about $10^{9} \mathrm{M}_{\odot}$ of atomic gas, at a mean velocity of about $7 \mathrm{~km} \mathrm{~s}^{-1}$. This is a measurable velocity, and would be present in existing data, though its effects are rather subtle in 
most cases. Lacey and Fall (1985) obtain somewhat lower velocities, some of which is the result of making up for the molecular gas depletion by infall as well as inflow and by having significantly higher star formation rates in the past. When account is made of the different assumptions used in their work and the estimates presented here, the estimated flow velocities are similar.

In any event, in order that the molecular gas not be depleted in a small fraction of a Hubble time, the molecular gas be replenished by inflow from the disk or infall from ouside the Galaxy if the current estimates of the star formation rate and gas return rates from stars are approximately correct. In addition, any inflow must be accompanied by angular momentum transfer and the size of the effects should be measurable.

\subsection{EXPONENTIAL DISKS}

We may now ask how long, at the current rate of star formation, would it take to make a disk with the current mass? If the local star formation rate is 3-7 $\times 10^{-9} \mathrm{M}_{\odot} \mathrm{yr}^{-1}$, and if the local disk surface density is $\sim 35 \mathrm{M}_{\odot} \mathrm{pc}^{-2}$ Gould (1996), then with a constant star formation rate, one can build up the disk of the Milky Way in 7-12 Gyr, a plausible amount of time. In other words, if the gas flows and conversion from atomic to molecular gas have both been in a steady state until today and if the efficiency of star formation has been unchanged, then the present exponential stellar disk of stars would have been built up over a Hubble time. The observational evidence is thus consistent with the idea that the stellar disk is exponential because the gas is exponential. On the other hand, the current observational evidence allows a range of past star formation rates and it is desirable to have tighter constraints on the star formation history of the disk. New data on stellar metallicities that might come from large scale photometric surveys, such as the OGLE, MACHO, EROS, and DUO microlensing searches as well as as the Sloan Digital Sky Survey, could produce valuable new data on this important question.

\section{Evidence for Inward Gas Motions}

\subsection{THE BAR}

Bars have long been argued to produce shocks in the gas within them causing inflows (e.g., Sanders and Huntley 1976). The supposition that the Milky Way is a barred galaxy has been around since 1964 (de Vaucouleurs) and has received strong confirmation in the last five years (see Blitz 1994 for a review). Dwek et al. (1994) have analyzed the COBE data, have made the first reliable modeling of the bar, and have concluded that the axial ratio 
of the bar is about $3 \frac{1}{2}: 1$. Evidently, the Milky Way is a strongly barred galaxy.

Binney et al. (1991) have made dynamical arguments for the existence of a bar based on the kinematics of the gas in the central regions of the Milky Way and the recognition that stable gas orbits must be non-intersecting. They showed that the observed gas distributions could be reasonably explained if there were a stellar bar with an extent of about 3-3.5 kpc. Inward of this radius, there are two families of non self-intersecting orbits that correspond to locations where gas could exist for relatively long periods of time. At other locations, the gas would rapidly shock and be driven inward, piling up at stable locations until other effects further change the radial gas distribution (Blitz et al. 1993).

If the bar is then responsible for the truncation of the exponential $\mathrm{CO}$ distribution in the Milky Way, similar effects should be seen in other barred galaxies. Indeed, millimeter interferometry has shown that barred galaxies always have a resolved central maximum similar to that seen in the Milky Way, often with complex structure, and a relatively empty region out to a location near the ends of the bar, which may or may not show secondary maxima (e.g., Kenney et al. 1992; Regan 1996). But if bars redistribute the angular momentum of the gas in the central regions of a galaxy, what would redistribute the angular momentum of the atomic gas in the outer regions of the disk, and what would produce a relative difference in $\mathrm{HI}$ vs. $\mathrm{H}_{2}$ flow velocities?

A related question that may be asked is whether the molecular gas seen at the Galactic center is simply the result of the the redistribution of the molecular gas inside the truncation radius by the bar. This is equivalent to asking whether the area under the point closest to the center in Figure 2 is equal to that under the fitted line inside $4 \mathrm{kpc}$ extrapolated to $\mathrm{R}=0$ $\mathrm{kpc}$. The answer is no. The $\mathrm{H}_{2}$ mass one would expect inside of $4 \mathrm{kpc}$ is 7 $\times 10^{8} \mathrm{M}_{\odot}$. The mass of the nuclear CO disk is $8 \times 10^{7} \mathrm{M}_{\odot}$ if the X-factor is the same both in the center and in the disk. If anything, changes in the $\mathrm{X}$-factor would imply lower masses in the center than what one infers from the CO emissivity alone (Blitz et al. 1986; Sodrowski et al. 1994).

\subsection{EVIDENCE FOR INFALL AND INFLOW}

If the star formation in the disk of the Milky Way is in or near a steady state, then one may ask, is there evidence for infall or inflow of HI to make up for the depletion of the molecular gas? There is in fact evidence for both, but it is ambiguous and may be attributable to other phenomena. The high velocity hydrogen clouds, for example (see Wakker 1993 for a review), and the low negative velocity gas observed toward both northern 
and southern galactic polar caps (Kulkarni \& Fich 1985) might both be manifestations of infalling gas. Evidence for inflow might be the negative $\mathrm{HI}$ and $\mathrm{CO}$ velocities relative to the stars seen in the Galactic anticenter (e.g., Metzger \& Schechter 1994). Further work is needed to disentangle a clear infall or inflow signature from other effects.

\section{References}

Bloemen, J.B.G.M., et al.1986, A\&A, 154, 25

Bieging, J. 1989, in The Evolution of the Interstellar Medium, L. Blitz, ed., ASP: San Francisco, 137

Binney, J. et al. 1991, MNRAS, 252, 210

Blitz, L. 1994, in Back to the Galaxy, S.S. Holt \& F. Verter, eds., AIP Press: New York, 98

Blitz, L., Bloemen, J.B.G.M., Hermsen, W., \& Bania, T.M. 1985, A\&A, 143, 267

Blitz, L., et al. 1993, Nature, 361, 417

Burton, W.B. \& Gordon, M.A. 1978, A\&A, 63, 7

Cohen, R.S. 1977, Ph.D. Dissertation, Columbia University

Dame, T. et al. 1987, ApJ, 322, 706

Dame, T. 1993, in Back to the Galaxy, S.S. Holt \& F. Verter, eds., AIP Press: New York, 267.

de Vaucouleurs, G. 1964, in The Galaxy and the Magellanic Clouds, eds. F.J. Kerr \& Rodgers, A.W., Australian Academy of Science: Sydney, 195

Güsten R., \& Mezger, P. 1983, Vistas Astr., 26, 159

Henderson, A.P., Jackson, P.D., \& Kerr, F.J. 1982, ApJ, 263116

Kenney, J., et al. 1992, ApJ, 395, L79

Knapp, G.R. 1991 in Frontiers of Stellar Evolution, D.L. Lambert ed., ASP: San Francisco, 229

Kulkarni, S.R., \& Fich, M. 1985, ApJ, 290, 792

Lacey, C.G. \& Fall, S.M. 1985 ApJ, 290, 154

Lyne, A., Manchester, R. \& Taylor J. 1985, MNRAS, 213, 613

Spergel, D.N., Malhotra, S., \& Blitz, L. 1996, in Spiral Galaxies in the Near-IR, ESOGarching, D. Minnitti \& H. Rix, eds., ESO-Garching, in press

Metzger, M.R., \& Schechter, P.L. 1994, ApJ, 420 , 177

Miller, G., \& Scalo, J. 1979, ApJS, 41, 513

Prantzos, N., \& Aubert O. 1995, A\&A, 302, 69

Regan, M. 1996, Ph. D. Dissertation, University of Maryland

Sanders, D.B., Solomon, P.M., \& Scoville, N.Z. 1984, ApJ, 276, 182.

Sanders, R.H. \& Huntley, J.M. 1976, ApJ, 209, 53

Schwartz, P.R., Wilson, W.J., \& Epstein, E.E. 1972, ApJ, 186, 529

Sodroski, T., et al. 1994, ApJ, 428, 638

Spergel, D.N., Malhotra, S., \& Blitz, L. 1996, in Spiral Galaxies in the Near-IR, D. Minnitti \& $\mathrm{H}$. Rix, eds. ESO-Garching, in press

Strong, A.W., \& Mattox, J. 1996, A\&A (Lett.), 308, 21

van Buren, D. 1985, ApJ, 294, 567

Wakker, B. 1993, Ph. D. Dissertation, University of Leiden

Wouterloot, J.G.A., Brand, J., Burton, W.B., \& Kwee, K.K. 1990, A\&A, 230, 21

Wilson, R.W., Jefferts, K.B. \& Penzias, A.A. 1970, ApJ, 161, L43

Wilson, W.J., et al. 1974, ApJ, 191, 357 